\title{
Endogenous L-carnitine levels and their correlation of lipid profiles between vegetarians and omnivores
}

\author{
Tsung-Jen Lin ${ }^{1}$, Sheau-Chung Tang ${ }^{2,3,4}$, Pei-Yun Liao ${ }^{2,4}$, Jen-Hung Yang ${ }^{2,3,5}$, Chin-Hung Liu ${ }^{1,6}$
}

${ }^{1}$ Institute of Pharmacology and Toxicology, Tzu Chi University, Taiwan, ${ }^{2}$ Department of Dermatology, Buddhist Tzu Chi General Hospital, Hualien, Taiwan, ${ }^{3}$ Department of Biochemistry, School of Medicine, Tzu Chi University, Hualien, Taiwan, ${ }^{4}$ Department of Research, Buddhist Tzu Chi General Hospital, Hualien, Taiwan, ${ }^{5}$ Institute of Medicine, School of Medicine, Tzu Chi University, Hualien, Taiwan, ${ }^{6}$ Department of Pharmacology, Tzu Chi University, Hualien, Taiwan

Background: Plant-based diet has been associated with lower risk of cardiovascular diseases (CVDs) and mortality, partly due to its effect on serum lipid profiles. L-carnitine (L-Car) is a conditionally essential nutrient that plays a vital role in energy production, carbohydrates and fatty acids metabolism. However, the levels of L-Car have less elucidated either in vegetarians and omnivores. This study aimed to compare the levels of L-Car and their correlation with blood glucose, lipid profiles and some CVDs risk factors (i.e. L-homocysteine, L-Hcy and trimethylamine N-oxide, TMAO) among vegetarians and omnivores.

Methods: A total of 56 healthy male (vegetarians, $n=9$; omnivores, $n=16$ ) and female (vegetarians, $n=15$; omnivores, $\mathrm{n}=16$ ) were recruited. Fasting blood samples were collected for biochemical testing and simultaneous analyzed of CVD biomarkers by using LC-MS.

Results: There was no significant difference in age (average 45 years old), body mass index, blood glucose, total cholesterol (TCH), triglyceride (TG) high-density lipoprotein cholesterol (HDL-C), methylmalonic acid (MMA), L-Hcy and TMAO among vegetarians and omnivores. The vegetarians had significantly higher concentration of L-Car $(\mathrm{p}=0.026)$ and ascorbic acid $(\mathrm{p}=0.010)$ than in omnivores. In parallel, the vegetarians had significantly lower low-density lipoprotein cholesterol (LDL-C, $\mathrm{p}=0.045)$ and 3-methylhistidine (3-MH, $\mathrm{p}=0.039)$ than in omnivores. Interestingly, LCar was demonstrated the negative correlation with TG $(\mathrm{r}=-0.4617, \mathrm{p}=0.040)$ and positive correlation with HDL-C $(\mathrm{r}=0.5680, \mathrm{p}=0.011)$ in vegetarians. In omnivores, L-Car was indicated the strong negative correlation with $\mathrm{TCH}(\mathrm{r}=$ -0.9524, $\mathrm{p}<0.001)$, LDL-C $(\mathrm{r}=-0.9236, \mathrm{p}<0.001)$ and positive correlation with blood glucose $(\mathrm{r}=0.7538, \mathrm{p}=0.004)$.

Conclusions: Higher levels of L-Car in the vegetarians might be associated with the higher endogenous L-Car than in omnivores as the compensation for lower carnitine levels obtained from the diet. Our results also suggested that endogenous L-Car might be playing as a CVDs protection factor both in vegetarians and omnivores through different influence on blood lipids. 